\title{
The First Presentation of Pediatric COVID-19 With Diabetic Ketoacidosis: A Unique Case Report
}

\author{
Shahin Jafarpoor ${ }^{1}$, Masoumeh Abedini ${ }^{1}$, Fatemeh Eghbal ${ }^{2}$, Amin Saburi ${ }^{2,3^{*}}$ \\ ${ }^{1}$ Department of Pediatrics, Faculty of Medicine, Kurdistan University of Medical Sciences, Sanandaj, Iran \\ ${ }^{2}$ Shahid Ghaazi General PolyClinic, Sanandaj, Iran \\ ${ }^{3}$ Chemical Injuries Research Center, Baqiayatalah University of Medical Sciences, Tehran, Iran
}

Corresponding Author: Amin Saburi, MD, Clinical Radiologist, Researcher, Chemical Injuries Research Center, Baqiyatallah University of Medical Sciences, Mollasadra St, VanakSq, Tehran, Iran. Tel: +98-9127376851,

Email: aminsaburi@yahoo.com

Received May 18, 2020; Accepted July 10, 2020; Online Published July 25, 2020

\begin{abstract}
Introduction: Severe acute respiratory syndrome coronavirus 2 (SARS-CoV-2), or COVID-19, is a pandemic infectious disease involved all over the world. Its mortality, especially in cases with additional co-morbidities, is so high that is has attracted the attention of the world. Diabetes mellitus is known as one of its risk factors for mortality.

Case Presentation: A unique case of pediatrics COVID-19 who presented with diabetic ketoacidosis (DKA) is reported in this manuscript as the first presentation. He presented with abdominal pain, nausea, and vomiting. RT-PCR test for COVID-19 via nasal swab was performed, and a positive diagnosis was obtained. Chest CT scan confirmed the diagnosis with multifocal bilateral patchy consolidation. Case was discharged after usual treatment of COVID-19.

Conclusion: In pediatrics, this disease can be subtle and have a confusion presentation, but it should be controlled to avoid spread of the disease. During epidemic status, suspicious cases specially children with nonspecific symptoms and other underlying diseases should be considered as at risk or potentially asymptomatic COVID-19 cases.

Keywords: Pediatrics, Coronavirus, Pneumonia, Diabetes Mellitus
\end{abstract}

Citation: Jafarpour S, Abedini M, Eghbal E, Saburi A. The first presentation of pediatric COVID-19 with diabetic ketoacidosis: a unique case report. Int J Travel Med Glob Health. 2020;8(3):131-133. doi:10.34172/ijtmgh.2020.22.

\section{Introduction}

Recent severe acute respiratory syndrome coronavirus 2 (SARS-COV2; COVID-19) has affected the whole world with a serious and fatal pandemic especially threatening to cases with comorbidities like diabetes. ${ }^{1,2}$ It is reported that the severity of the diseases, mortality, and further complications are more common in diabetic patients. Many considerations related to diabetic patients in SARS-Cov2 epidemic conditions, including the health requirements necessary for healthcare in the community and blood sugar control have been mentioned. ${ }^{1-3}$ One point of discussion is the possible complications in diabetic patients, especially children, which will be mention in the following case. Herein is reported a unique case of COVID-19; to the best of our knowledge, this is the first case reported concerning this topic.

\section{Case Presentation}

A 9-year-old boy, $20 \mathrm{~kg}$ in weight, and with a previous history of poorly controlled diabetes mellitus type 1 (DM), was referred to the local hospital. He presented with abdominal pain, nausea, and vomiting. After a diagnosis of acute appendicitis, he underwent appendectomy. The laboratory findings at admission were within normal range except for blood sugar (BS): $180 \mathrm{mg} / \mathrm{dL}$ and with blood cell (WBC) count: 12000/ $\mu \mathrm{L}$, polymorphonuclear $(\mathrm{PMN}): 95 \%$. Ultrasound assessment revealed a fixed blind bowel loop with normal diameter (4.5 $\mathrm{mm}$ ) in the right lower quadrant.

Post operation, BS increased (after 8 hours) and symptoms of restlessness, weakness, lethargy, and cough were observed in the patient. The patient was referred to our center as a tertiary referral pediatrics hospital with a diagnosis of diabetic ketoacidosis (DKA) according to the following laboratory findings: $\mathrm{pH}$ : 7.2, PCO2: $26 \mathrm{~mm} \mathrm{Hg}$, HCO3: 16 mmol/dL, ketonuria: 3+, fasting blood sugar (FBS): $400 \mathrm{mg} /$ dL. Post-appendectomy complications were excluded due to the administration of suitable antibiotic therapy and a normal

Copyright $(\odot 2020$ The Author(s). This is an open-access article distributed under the terms of the Creative Commons Attribution License (http:// creativecommons.org/licenses/by/4.0), which permits unrestricted use, distribution, and reproduction in any medium, provided the original work is properly cited. 


\section{ultrasound.}

There were some suspicions indicating an underlying diagnosis that made the patient susceptible to DKA. First, frequent nonproductive cough, mild dyspnea, and tachypnea for 3 days, continuous uncontrolled FBS (exactly before appendectomy equal to $430 \mathrm{mg} / \mathrm{dL}$ ), and decreased $\mathrm{O} 2$ saturation and its changes with or without oxygen therapy (94\% vs $84 \%$ ). Because of the COVID-19 epidemic, the subject underwent additional chest computed tomography (CT), which revealed bilateral multifocal patchy groundglass opacities in the lower lung lobes (Figure 1). RT-PCR test for COVID-19 via nasal swab was performed, and a positive diagnosis was obtained. The subject underwent treatment with hydroxychloroquine and Kaletra (containing a combination of lopinavir and ritonavir) for COVID-19 and insulin and serum therapy and potassium for DKA, and his status became stable.

\section{Discussion}

Since December 2019 when COVID-19 was recognized and rapidly became a pandemic disease, many reports have been published about the clinical/paraclinical manifestations in pediatrics that were deferent to adult COVID-19.1,2 Many aspects of COVID-19 were considered in children; it is more likely to be asymptomatic, and laboratory, clinical, and radiological results show more unusual findings than in the adult population. ${ }^{3,4}$ Severe forms of the disease have been reported less frequently in children. ${ }^{5}$ Based on our experience, some considerations can be suggested in this case.

First, COVID-19 can be asymptomatic or sub-clinically symptomatic. In pediatrics, COVID-19 may present with nonspecific symptoms such as abdominal and gastrointestinal

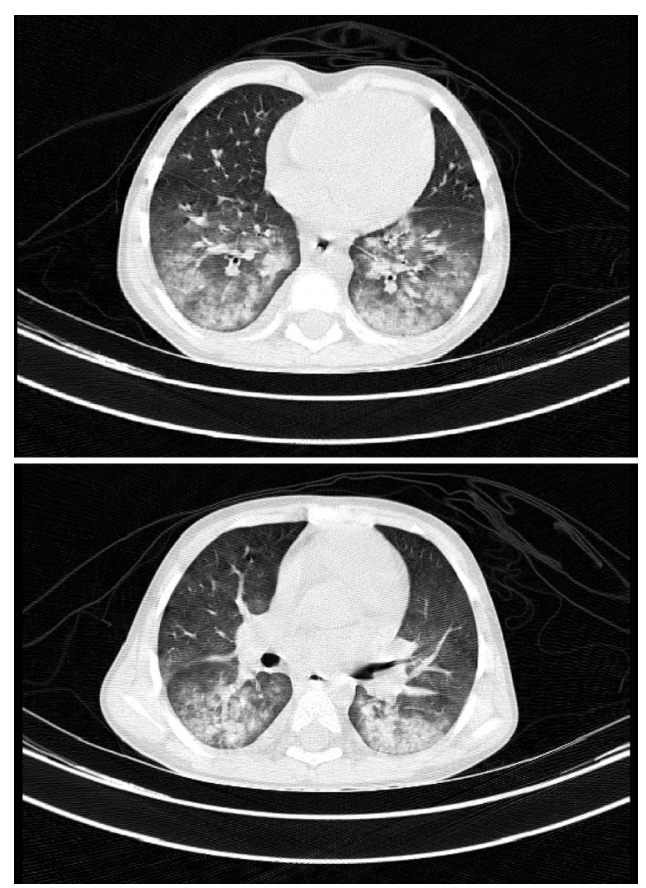

Figure 1. Non-enhanced chest CT showing ground-glass opacities with sub-pleural sparing in the lower lobes bilaterally, typically reported in COVID-19. symptoms, and these asymptomatic cases may infect other people, such as general healthcare personnel. Second, COVID-19 may trigger or worsen underlying diseases in pediatric patients such as uncontrolled DM or metabolic disorders as previously reported for the H1N1 influenza. ${ }^{6}$ Third, in affected regions, any suspicious symptoms like subtle dyspnea, low grade fever, or unlocalized abdominal pain should be subject to further evaluation. ${ }^{7}$ Fourth, chest CT scan should be performed in suspicious cases. Fifth, patients' physical conditions and responses to the disease can be quite different from those of others, even those of similar age and physical status. It may depend on cultural situation, incomplete and inconclusive previous treatment, host immunologic response, etc. Sixth, viral infection and its immunologic response can trigger DM onset as reported for SARS. ${ }^{8}$ Due to further referral of diabetic cases to clinics and hospitals as well as the relative immune deficiency of such patients, there is an increased risk of infection. ${ }^{9}$

\section{Conclusion}

It was suggested that during epidemic status, suspicious cases with nonspecific symptoms and other underlying diseases should be considered as at risk or potentially asymptomatic COVID-19 cases.

\section{Authors' Contributions}

All authors have contributed equally to data collection, text writing, and final editing.

\section{Conflict of Interest Disclosures}

The authors declare no conflicts of interest regarding this work.

\section{Ethical Approval}

All data was duly presented, and all necessary interventional procedures were done. Moreover, written informed consent was obtained from the subject's parents.

\section{Funding/Support \\ None.}

\section{Acknowledgments}

The authors would like to acknowledge the personnel of the pediatric ward of Be'sat hospital, Sanandaj, Kurdistan, Iran as well as medical service staff around the world for their courage, dedication, self-sacrifice, and efforts to fight COVID-19.

\section{References}

1. Hong H, Wang Y, Chung HT, Chen CJ. Clinical characteristics of novel coronavirus disease 2019 (COVID-19) in newborns, infants and children. Pediatr Neonatol. 2020;61(2):131-132. doi:10.1016/j.pedneo.2020.03.001.

2. Chen N, Zhou M, Dong $X$, et al. Epidemiological and clinical characteristics of 99 cases of 2019 novel coronavirus pneumonia in Wuhan, China: a descriptive study. Lancet. 2020;395(10223):507513. doi:10.1016/S0140-6736(20)30211-7.

3. Li W, Cui H, Li K, Fang Y, Li S. Chest computed tomography in 
children with COVID-19 respiratory infection. Pediatr Radiol. 2020;50(6):796-799. doi:10.1007/s00247-020-04656-7

4. Sun D, Li H, Lu XX, et al. Clinical features of severe pediatric patients with coronavirus disease 2019 in Wuhan: a single center's observational study. World J Pediatr. 2020;16(3):251-259. doi:10.1007/s12519-020-00354-4.

5. Wu C, Chen X, Cai Y, et al. Risk Factors Associated With Acute Respiratory Distress Syndrome and Death in Patients With Coronavirus Disease 2019 Pneumonia in Wuhan, China. JAMA Intern Med. 2020;180(7):1-11. doi:10.1001/ jamainternmed.2020.0994.

6. Tan H, Wang C, Yu Y. H1N1 influenza: the trigger of diabetic ketoacidosis in a young woman with ketosis-prone diabetes. Am J Med Sci. 2012;343(2):180-183. doi:10.1097/ MAJ.0b013e3182376cc4

7. Wong SH, Lui RN, Sung JJ. Covid-19 and the digestive system. J Gastroenterol Hepatol. 2020;35(5):744-748. doi:10.1111/ jgh.15047

8. Yang JK, Lin SS, Ji XJ, Guo LM. Binding of SARS coronavirus to its receptor damages islets and causes acute diabetes. Acta Diabetol. 2010;47(3):193-199. doi:10.1007/s00592-009-0109-4

9. Groups at Higher Risk for Severe Illness. Available from: https:// www.cdc.gov/coronavirus/2019-ncov/need-extra-precautions/ groups-at-higher-risk.html 